Introduction Euro-GASP antimicrobial resistance (AMR) data informs gonorrhoea (GC) treatment guidelines, therefore the data needs to be robust and reliable. We assessed whether Euro-GASP appropriately reflects the AMR situation across Europe.

Methods We compared data from Euro-GASP and national systems from 12 countries for azithromycin (AZ), cefixime (CEF) and ciprofloxacin (CIP) from 2009-2013 and performed Poisson regression to identify differences $(p<0.05)$ between the proportions of resistant isolates in Euro-GASP and national data sets. The 2014 Euro-GASP AMR data for each country $(n=19)$ were weighted to account for differences according to patient characteristics (age group, sexual orientation and gender) between the Euro-GASP patient data and the European STI surveillance data. Data were compared to determine whether resistance levels shifted above or below the $5 \%$ threshold used to assess the clinical utility of GC treatments.

Results Euro-GASP isolates were more likely to be resistant for AZ (incidence-rate ratio (IRR) 1.3, 1.1-1.5 95\% confidence interval $(\mathrm{CI}), \mathrm{p}=0.003)$ and less likely to be resistant for CEF (IRR 0.8, 0.7-0.9 95\% CI, $\mathrm{p}=0.007$ ) compared to the national data. There was no significant difference for CIP.

Weighting slightly altered the estimates of overall AMR (-4.7\%-4.7\% difference). More apparent differences for AZ and CIP (9.5\%-17.9\%) were observed for countries with low isolate numbers and low completeness of reporting $(n=3)$. Weighting caused AMR levels to cross the 5\% threshold in one country (CEF $5 \%$ to $3.4 \%$ ).

Conclusion The comparability of resistance proportions was satisfactory for CIP; MICs for CIP-resistant gonococci were mostly well above the resistance breakpoint, so testing and interpretation remained reliable. The differences for CEF and AZ resistance proportions were likely due to variability induced by strains with MICs close to the resistance breakpoints. Weighting of the Euro-GASP 2014 data provided similar estimates of AMR, demonstrating that Euro-GASP laboratories submit representative isolates in respect to patient characteristics.

\section{P3.158 DISPPARITIES IN HIV CLINICAL OUTCOMES AMONG A LARGE COHORT OF HIV-INFECTED PERSONS IN CARE IN MISSISSIPPI, UNITED STATES: FINDINGS FROM MEDICAL MONITORING PROJECT, 2009-2014}

${ }^{1}$ Mina Qobadi, ${ }^{1}$ Ali Dehghanifirouzabadi, ${ }^{2}$ Tiffany Mcdonald, ${ }^{1}$ Tametria Samms, ${ }^{1}$ Kendra Johnson. ${ }^{1}$ Mississippi State Department of Health, Jackson, USA; ${ }^{2}$ Mississippi State Department of Health, Jacskon, USA

\subsection{6/sextrans-2017-053264.393}

Introduction Increase in CD4 count and achieving viral suppression are the ultimate goals of the HIV care, yet disparities in these outcomes exist among subpopulations of HIV-infected persons. We aimed to assess potential disparities in viral suppression and normal CD4 count among HIV-infected persons in care in Mississippi.

Methods We used Mississippi Medical Monitoring Project (MMP) data from 2009 to $2014(n=1,233)$ in this study. MMP is a nationally representative surveillance system designed to assess and monitor the behavioural and clinical characteristics of HIV-infected adults receiving outpatient medical care in the United States. Outcome variables in this study were suppressed recent and durable viral load $(<200$ copies/ $\mathrm{ml})$, and normal CD4 count $\left(\geq 500\right.$ cells $\left./ \mathrm{mm}^{3}\right)$. Patients' characteristics in this study were race, gender, age, annual income, education, insurance, and length of diagnosis. Descriptive analysis, Chi-square tests, and multiple logistic regression were conducted, accounting for the complex sample design.

Results Our findings indicate that suppressed recent viral load prevalence was significantly higher among whites (72.8\%), those aged $\geq 50$ years $(74.3 \%)$, those who had annual income $\geq \$ 20000$ (76\%), public insurance (68.3\%), or those were diagnosed $\geq 5$ years (67.4\%). The prevalence of suppressed durable viral load was significantly higher among those who aged $\geq 50$ years $(71.7 \%)$, and were diagnosed $\geq 5$ years ago (61.4\%), and the prevalence of normal CD4 was significantly higher among females (46\%). Multivariable analysis show that those aged $\geq 50$ years were more likely to have suppressed recent $(\mathrm{aOR}=2.4)$ and durable viral load $(\mathrm{aOR}=2.9)$ compared to those aged 18-24 years, and women were more likely to have normal $\mathrm{CD} 4$ count than men $(\mathrm{aOR}=1.4)$, after controlling for the confounders.

Conclusion Our findings identified age and gender disparities in the HIV clinical outcomes, which may be used to develop and implement multifaceted interventions to improve health equity among all HIV-infected patients.

\section{P3.159 PREVALENCE OF SEXUALLY TRANSMITTED INFECTIONS AND ACCEPTABILITY, FEASIBILITY OF SCREENING IN ANTENATAL CARE, VIETNAM, 2016-2017}

${ }^{1}$ Minh Nguyen, 'Le Minh Giang, ${ }^{1}$ Nguyen Duc Hinh, ${ }^{2}$ Jeffrey Klausner. ${ }^{1}$ Hanoi Medical University, Hanoi - North Vietnam; ${ }^{2}$ University of California, Los Angeles, Los Angeles, USA

\subsection{6/sextrans-2017-053264.394}

Introduction There is strong evidence that sexually transmitted infections (STIs) cause adverse pregnancy outcomes, including preterm birth, stillbirth, low birth-weight and congenital infections. The prevalence of Chlamydia trachomatis (CT), Neisseria gonorrhoeae (NG) and Trichomonas vaginalis (TV) in the Asia Pacific region varies. The recently estimated mean prevalence of CT, NG and TV in low and middle income countries in Asia was $0.8 \%, 2.8 \%$ and $13.6 \%$ respectively. In Vietnam, pregnant women are not screened for CT, NG and TV during their antenatal visits. The aim of our current study was to estimate the prevalence of STIs and access feasibility and acceptability of screening for CT, NG and TV among pregnant women in Hanoi.

Methods We plan to enrol 800 pregnant women coming to Ha Dong General Hospital, Hanoi for antenatal care. Eligibility includes age 18 years or older, gestational age less than 35 weeks and willing to come back to the hospital for counselling and treatment if tested positive. After informed consent, enrolled women self-collect vaginal swabs or urine samples for testing using Xpert CT/NG and TV assays (Cepheid, Sunnyvale, CA, USA). We offer CT/NG testing to all 800 participants but only offer TV testing to the first 387 participants (due to TV's low prevalence).

Results To date we have enrolled 490 participants (Acceptability was 99.0\% (95\%CI, 97.7-99.7)). Among them, 34 had CT (6.9\%; 95\% CI, 4.9-9.6) and none had NG. Among 387 women who were tested for TV, 3 had the infection $(0.78 \%$; 95\% CI, 0.16-2.25). Feasibility was 94.4\% (95\%CI, 81.399.3), with 34 out of 36 infected women treated for the corresponding infection. 